\title{
ANNE PlouviER-DEBAIGT \\ Solutions renormalisées pour des équations autonomes des milieux poreux
}

\author{
Annales de la faculté des sciences de Toulouse $6^{e}$ série, tome $6, \mathrm{n}^{\circ} 4$ \\ (1997), p. 727-743 \\ <http://www.numdam.org/item?id=AFST_1997_6_6_4_727_0>
}

(C) Université Paul Sabatier, 1997, tous droits réservés.

L'accès aux archives de la revue «Annales de la faculté des sciences de Toulouse » (http://picard.ups-tlse.fr/ annales/) implique l'accord avec les conditions générales d'utilisation (http://www.numdam.org/conditions). Toute utilisation commerciale ou impression systématique est constitutive d'une infraction pénale. Toute copie ou impression de ce fichier doit contenir la présente mention de copyright.

\section{NumDam}

Article numérisé dans le cadre du programme Numérisation de documents anciens mathématiques http://www.numdam.org/ 


\title{
Solutions renormalisées pour des équations autonomes des milieux poreux ${ }^{(*)}$
}

\author{
Anne Plouvier-Debaigt ${ }^{(1)}$
}

\begin{abstract}
RÉsuMÉ. - On établit un résultat d'unicité pour les solutions faibles d'équations paraboliques quasi linéaires dégénérées des milieux poreux, lorsque le tenseur de diffusivité dépend de l'état instantané du système physique. Pour cela, on prouve que toute solution faible est une solution renormalisée, selon un critère qui discrimine les régions d'hyperbolicité et de parabolicité de la solution.

Abstract. - An uniqueness result is proved for the weak solutions of parabolic degenerate equations when the instantaneous state of the physical system is taken into account in the diffusivity tensor. Any weak solution is proved to be a renormalized one, according to admissibility criteria in the hyperbolicity and parabolicity free areas.
\end{abstract}

\section{Introduction}

On s'intéresse à l'étude analytique d'un problème d'évolution du type de la diffusion non linéaire en milieu poreux en prenant en compte l'influence de l'état instantané du système physique sur le tenseur de diffusivité.

On considère une classe d'équations non linéaires de type divergentiel

$$
\frac{\partial u}{\partial t}-\operatorname{div}(\mathcal{A}(\cdot, u) \nabla \phi(u))=0, \quad \phi(0)=\phi^{\prime}(0), \phi^{\prime} \geq 0,
$$

dans un cylindre $Q=] 0, T[\times \Omega$, associées à une condition de bord de type Neumann sur la frontière latérale $] 0, T[\times \partial \Omega$ et à une condition de Cauchy sur $\{0\} \times \Omega$.

(*) Reçu le 29 septembre 1995

(1) Université de Pau et des Pays de l'Adour, Laboratoire de Mathématiques Appliquées; U.P.R.E.S.-A C.N.R.S. 5033, I.P.R.A., Avenue de l'Université, F-64000 Pau (France) 
On établit que le problème est mathématiquement bien posé pour une donnée initiale a priori peu régulière et sans hypothèse liant le comportement local des fonctions d'état en limitant fortement l'ensemble admissible des lois phénoménologiques ou expérimentales, c'est-à-dire on présente un résultat d'existence et principalement d'unicité (c'est ici le point réellement délicat) sous les hypothèses "naturelles" rencontrées en pratique pour l'étude des migrations de fluides en milieu poreux. La démonstration se fonde sur l'observation que toute solution faible du problème est une solution renormalisée selon un critère qui discrimine les régions d'hyperbolicité et de parabolicité de la solution avec un contrôle de l'énergie près des régions où l'équation est dégénérée, au sens de J.-L. Lions.

Ce problème modélise en particulier la diffusion d'un gaz dans un milieu poreux représenté par $\Omega$, de porosité constante et égale à 1 après un classique changement d'échelle des temps par homothétie. $\grave{A}$ titre d'illustration, désignant par $\rho$ la masse volumique du gaz, $\vec{U}$ son vecteur de vitesse de filtration, on écrit l'équation de conservation de masse (équation de continuité)

$$
\frac{\partial \rho}{\partial t}+\operatorname{div}(\rho \vec{U})=0 \quad \operatorname{dans} Q
$$

On adopte pour lois de comportement :

(i) une loi de type Boyle-Mariotte

$$
\begin{cases}\rho(0)=0 & \\ \rho=\rho(P) & \rho \text { fonction strictement croissante } \\ & 0 \leq \rho \leq \rho_{\max }<+\infty\end{cases}
$$

liant la masse volumique et la pression (exemple, $\rho=c P^{1 / \gamma}(\gamma=1,4$ pour l'air), $P$ admissible, c'est-à-dire $P \in[0, \bar{P}]$ avec un prolongement ad hoc hors du domaine usuel);

(ii) une loi de Darcy non linéaire, donnant le vecteur $\vec{U}$ en fonction du gradient de pression $\nabla P$ selon la formule :

$$
\vec{U}=-\mathcal{K}(x, P) \cdot \nabla P
$$

où l'on désigne par $\mathcal{K}$ le tenseur de diffusivité.

Ceci conduit à une équation du type parabolique non linéaire dégénérée :

$$
\frac{\partial S}{\partial t}-\operatorname{div}(\mathcal{A}(x, S) \nabla \phi(S))=0, \quad \phi(0)=\phi^{\prime}(0), \phi^{\prime} \geq 0,
$$


Solutions renormalisées pour des équations autonomes des milieux poreux

en prenant pour inconnue $S=\rho(P)$, et pour fonctions auxiliaires

$$
\phi(S)=\int_{0}^{\rho^{-1}(S)} \rho(r) \mathrm{d} r, \quad \mathcal{A}(x, S)=\mathcal{K}\left(x, \rho^{-1}(S)\right) .
$$

L'imperméabilité de $\partial \Omega=\Gamma$ s'exprime par la condition de Neumann homogène :

$$
(\mathcal{A}(x, S) \nabla \phi(S)) \cdot \vec{n}=0 \quad \text { sur }] 0, T[\times \Gamma \text {. }
$$

\section{1. Énoncé de la formulation variationnelle}

\section{a) Notations générales}

On désigne par $\mathcal{L}^{p}$ la mesure de Lebesgue sur $\mathbb{R}^{p}$, pour tout entier naturel $p$ et $\mathcal{H}^{p-1}$ la mesure $(p-1)$-dimensionnelle de Hausdorff.

Soit $\Omega$ un domaine borné de $\mathbb{R}^{N}, N \in \mathbb{N}^{*}$ de frontière $\Gamma$ lipschitzienne et de vecteur unitaire extérieur $\vec{n}$, défini $\mathcal{H}^{N-1}$ presque partout sur $\partial \Omega ; T$ désignant un réel strictement positif, on note $Q=] 0, T[\times \Omega$.

On se donne, pour $(i, j) \in(1, \ldots, N)^{2}, N^{2}$ fonctions de Carathéodory :

$$
\begin{aligned}
a_{i j}: \Omega \times \mathbb{R}^{+} & \longrightarrow \mathbb{R} \\
(x, \lambda) & \longrightarrow a_{i j}(x, \lambda),
\end{aligned}
$$

vérifiant l'hypothèse $(\mathrm{H} 1)$ :

(i) $\exists \ell>0$,

$$
\left\|a_{i j}\right\|_{L^{\infty}(\Omega \times \mathbb{R}+)} \leq \ell
$$

pour tout $(i, j) \in(1, \ldots, N)^{2}$

(ii) $\exists \alpha_{0}>0, \forall \lambda \in \mathbb{R}^{+}, \forall \xi \in \mathbb{R}^{N}$,

$$
a_{i j}(\cdot, \lambda) \xi_{i} \xi_{j} \geq \alpha_{0} \xi_{i} \xi_{i} \quad \mathcal{L}^{N} \text {-p.p. sur } \Omega
$$

(avec la convention de sommation de l'indice répété);

(iii) pour tout $(i, j) \in(1, \ldots, N)^{2}, \forall \lambda \in \mathbb{R}^{+}$,

$$
y \longrightarrow a_{i j}(x, y)
$$

est lipschitzienne uniformément par rapport à $x, \mathcal{L}^{N}$-p.p. dans $\Omega$. 
On note $\mathcal{A}(x, \lambda)$ la matrice de diffusivité de coefficient générique $\left\{a_{i j}(x, \lambda)\right\}_{N \times N}$.

\section{b) Cadre fonctionnel de la modélisation}

On introduit l'espace de Hilbert $V=H^{1}(\Omega)$ muni de sa norme usuelle.

Identifiant $H=L^{2}(\Omega)$ à son dual, on peut identifier $V^{\prime}$, le dual de $V$, à un sur-espace de $H$, avec $V \hookrightarrow H \hookrightarrow V^{\prime}$, l'injection de $V$ dans $H$ étant continue, à image dense.

On note $\langle\cdot, \cdot\rangle_{V^{\prime}, V}$ la dualité $V^{\prime}, V$.

Hypothèses sur $u_{0}$ et $\phi$

Comme dans [9] et [10], on s'intéresse essentiellement à la recherche de solutions bornées et positives, associées à une donnée initiale bornée, positive sans autre hypothèse de régularité a priori. On suppose donc:

(H2) $u_{0} \in L^{\infty}(\Omega), 0 \leq u_{0} \leq 1 \mathcal{L}^{N}$-presque partout dans $\Omega$;

(H3) $\phi \in C^{1}([0,1]), \phi(0)=\phi^{\prime}(0)=0, \forall r>0, \phi^{\prime}(r)>0$,

$\phi^{\prime}$ localement lipschitzienne sur $\left.] 0,1\right]$,

$\phi^{-1} \in \mathcal{C}^{0, \alpha}([0, \phi(1)])$, c'est-à-dire $\phi^{-1}$ est höldérienne d'ordre $\alpha$, pour un certain $\alpha \in] 0,1[$, sur l'intervalle $[0, \phi(1)]$.

Dans la pratique, on considère généralement pour $r \in[0,1], \phi(r)=r^{m}$ avec $m>1$. On introduit alors le problème de Cauchy, formellement énoncé par la formulation variationnelle suivante, qui tient compte d'une condition de bord de Neumann homogène sur $\Gamma$,

$$
\begin{gathered}
\frac{\partial}{\partial n_{A}} \phi(u)=(\mathcal{A}(\cdot, u) \nabla \phi(u)) \cdot \vec{n}=0, \\
\left\{\begin{array}{l}
\text { trouver } u \text { vérifiant, presque partout sur }] 0, T[ \\
\forall v \in V, \quad\left\langle\frac{\partial u}{\partial t}, v\right\rangle_{V^{\prime}, V}+\int_{\Omega} \mathcal{A}(x, u) \nabla \phi(u) \cdot \nabla v \mathrm{~d} x=0, \\
u(0)=u_{0} \mathcal{L}^{N} \text {-p.p. dans } \Omega .
\end{array}\right.
\end{gathered}
$$

\section{Existence d'une solution faible au problème $(P)$}

Grâce au lemme technique d'intégration de F. Mignot et A. Bamberger [3] et à une étude préalable de M. Artola [2] dans le cadre particulier où $\phi=\mathrm{Id}$, on établit le résultat d'existence suivant. 
Proposition 2.1.- Sous les hypothèses (H1), (H2) et (H3), il existe au moins une solution $u$ du problème $(P)$ telle que :

$$
\left\{\begin{array}{l}
u \in L^{\infty}(Q), 0 \leq u \leq 1 \mathcal{L}^{N+1} \text {-p.p.dans } Q, \\
\phi(u) \in L^{2}\left(0, T ; H^{1}(\Omega)\right), \quad \frac{\partial u}{\partial t} \in L^{2}\left(0, T ;\left(H^{1}(\Omega)\right)^{\prime}\right), \\
u(0)=u_{0} \mathcal{L}^{N} \text {-p.p. dans } \Omega, L^{1}(\Omega)-\lim _{t \rightarrow 0^{+}} u(t, \cdot)=u_{0},
\end{array}\right.
$$

vérifiant, presque partout sur ]0, $T$ [, l'équation variationnelle

$$
\forall v \in H^{1}(\Omega), \quad\left\langle\frac{\partial u}{\partial t}, v\right\rangle_{V^{\prime}, V}+\int_{\Omega} \mathcal{A}(x, u) \nabla \phi(u) \cdot \nabla v \mathrm{~d} x=0 .
$$

La démonstration ne fait pas difficulté et reprend l'argumentation décrite dans ([9], [10]) : il s'agit essentiellement de régulariser le terme de diffusion par une classique méthode de viscosité artificielle, de prouver l'existence d'une solution visqueuse par le théorème de point fixe de Schauder dans le cadre hilbertien séparable et de s'affranchir de la viscosité artificielle par des arguments de compacité.

\section{Un résultat d'unicité}

En imposant une condition supplémentaire d'höldérianité d'exposant supérieur ou égal à $1 / 2$ sur la dépendance $w \rightarrow\left\|\mathcal{A}\left(\cdot, \phi^{-1}(w)\right)\right\|$, il a été établi, notamment dans [1] et sa bibliographie et dans [8], un résultat d'unicité. Cependant, lorsque $\phi(r)$ est pris égal à $r^{m}, m>2$, ce résultat n'est plus applicable et la question de l'unicité reste posée, notamment dans le cas classique $m=3$.

On s'intéresse ici à obtenir l'unicité de la solution sans avoir recours à l'existence d'un module de continuité reliant les fonctions d'état $\left(a_{i j}\right)_{i, j=1, \ldots, N}$ et $\phi^{-1}$, c'est-à-dire sans condition académique sur le comportement local comparé des diverses fonctions d'état, qui au demeurant, décrivent des phénomènes physiques indépendants les uns des autres. Ainsi, sous la seule considération que, $\forall(i, j) \in\{1, \ldots, N\}^{2}$, l'application $\lambda \rightarrow a_{i j}(\cdot, \lambda)$ est lipschitzienne, via les travaux de D. Blanchard et H. Redwane [4], F. Murat [8] et de L. Boccardo, D. Giachetti, J. I. Díaz et F. Murat [5]. Selon les idées de R. J. Di Perna et P.-L. Lions [6], on établit l'unicité de la solution dite renormalisée en un sens que l'on va préciser, et, de fait, 


\section{Anne Plouvier-Debaigt}

de la solution du problème $(\mathrm{P})$, en prouvant que toute solution du problème $(\mathrm{P})$ est une solution renormalisée.

On dispose de la proposition suivante.

Proposition 3.1. - Sous les hypothèses (H1), (H2) et (H3), la solution $u$ du problème $(P)$ décrite par la proposition 2.1 est unique et possède en outre la propriété de régularité :

$$
u \in \mathcal{C}^{0}\left([0, T] ; L^{1}(\Omega)\right)
$$

Avant de démontrer ce résultat, on va, dans un premier temps, donner une formulation équivalente à celle du problème $(\mathrm{P})$ afin de définir la notion de solution renormalisée.

On introduit, pour cela, la suite de fonctions numériques $\left(S_{n}\right)_{n \in \mathbb{N}^{*}}$ définies sur $[0,1]$, telles que

$$
\left\{\begin{array}{l}
S_{n}(0)=0 \\
\forall r \in[0,1], \quad S_{n}^{\prime}(r)=\min \left((2 n r-1)^{+}, 1\right),
\end{array}\right.
$$

en vue de fournir une approximation à support dans ] 0,1$]$ de la fonction $\operatorname{Id}_{[0,1]}$. On peut facilement vérifier que la formulation du problème $(P)$. est équivalente à la formulation du problème $\left(\mathrm{P}^{*}\right)$ énoncé ci-dessous.

$$
\left\{\begin{array}{l}
u \in L^{\infty}(Q), 0 \leq u \leq 1 \mathcal{L}^{N+1} \text {-p.p. dans } Q, \\
\phi(u) \in L^{2}\left(0, T ; H^{1}(\Omega)\right), \quad \frac{\partial u}{\partial t} \in L^{2}\left(0, T ;\left(H^{1}(\Omega)\right)^{\prime}\right), \\
u(0)=u_{0} \mathcal{L}^{N} \text {-p.p. dans } \Omega, L^{1}(\Omega) \text { - } \lim _{t \rightarrow 0+} u(t, \cdot)=u_{0}, \\
\text { pour toute fonction } S \text { définie dans }[0,1], \text { convexe, nulle en } 0, \\
\text { telle que } \left.\left.S^{\prime} \in W^{1,+\infty}(] 0,1[) \text { avec supp } S^{\prime} \subset\right] 0,1\right], \text { on a, } \\
\left.\forall w \in L^{\infty}(\Omega) \cap H^{1}(\Omega) \text { et presque partout sur }\right] 0, T[, \\
\left\langle\frac{\partial u}{\partial t}, S^{\prime}(u) w\right\rangle_{V^{\prime}, V}+ \\
\quad+\int_{\Omega}\{\mathcal{A}(x, u) \nabla \phi(u)\} \cdot\left\{w \nabla S^{\prime}(u)+S^{\prime}(u) \nabla \omega\right\} \mathrm{d} x=0
\end{array}\right.
$$

et associée à la relation suivante :

$$
\lim _{n \rightarrow+\infty} \int_{Q}\{\mathcal{A}(x, u) \nabla \phi(u)\} \cdot \nabla S_{n}^{\prime}(u) \mathrm{d} x \mathrm{~d} t=0 .
$$


Solutions renormalisées pour des équations autonomes des milieux poreux

On va, à titre d'illustration, seulement démontrer la propriété (L).

Tout d'abord, on justifie le fait que $\forall n \in \mathbb{N}^{*}$, et pour presque tout $t$, $S_{n}^{\prime}(u(t)) \in H^{1}(\Omega)$. On peut noter que, $\forall n \in \mathbb{N}^{*}$, on a pour presque tout $t$,

$$
S_{n}^{\prime}(u(t))=S_{n}^{\prime} \widetilde{\circ \phi^{-1}}\left(\max \left(\phi\left(\frac{1}{2 n}\right), \phi(u(t))\right)\right) \mathcal{L}^{N} \text {-p.p. dans } \Omega
$$

où $S_{n}^{\prime} \widetilde{\circ \phi}^{-1}$ désigne la restriction de $S_{n}^{\prime} \circ \phi^{-1}$ sur l'intervalle $[\phi(1 / 2 n), \phi(1)]$. Or $S_{n}^{\prime} \widetilde{\circ \phi}^{-1}$ est définie sur $[\phi(1 / 2 n), \phi(1)]$ et lipschitzienne comme composée de fonctions lipschitziennes (puisque sur $[1 / 2 n, 1], \phi^{\prime}$ est minorée par une constante strictement positive, d'après (H3)). D'où, d'après le lemme de G. Stampacchia, $S_{n}^{\prime}(u(t)) \in H^{1}(\Omega)$ presque partout en $t$. Plus généralement, la propriété d'appartenance de $S^{\prime}(u(t))$ à $H^{1}(\Omega)$ pour presque tout $t$, essentielle pour la méthode, résulte de la condition imposée sur le support de $S^{\prime}$.

Il est donc loisible, d'après ce qui précède, de prendre comme fonctiontest, dans l'équation du problème $(\mathrm{P}), v=S_{n}^{\prime}(u)$. Il vient ainsi :

$$
\int_{Q}\{\mathcal{A}(x, u) \nabla \phi(u)\} \cdot \nabla S_{n}^{\prime}(u) \mathrm{d} x \mathrm{~d} t=-\int_{0}^{T}\left\langle\frac{\partial u}{\partial t}, S_{n}^{\prime}(u)\right\rangle_{V^{\prime}, V} \mathrm{~d} t .
$$

L'application définie par $r \rightarrow S_{n}^{\prime}(r)$ étant croissante (propriété commune à toutes les fonctions admissibles $S$, supposées convexes dans $W^{2,+\infty}(] 0,1[)$, on peut appliquer le lemme d'intégration de F. Mignot [3]. Il s'ensuit que

$$
\begin{aligned}
\int_{Q}\{\mathcal{A}(x, u) \nabla \phi(u)\} \cdot \nabla S_{n}^{\prime}(u) \mathrm{d} x \mathrm{~d} t & =-\int_{\Omega}\left(\int_{u_{0}(x)}^{u(T, x)} S_{n}^{\prime}(r) \mathrm{d} r\right) \mathrm{d} x \\
& =-\int_{\Omega}\left(S_{n}(u(T, x))-S_{n}\left(u_{0}(x)\right)\right) \mathrm{d} x .
\end{aligned}
$$

Il suffit maintenant de passer à la limite, quand $n \rightarrow+\infty$, ce qui est licite en observant que, d'après le théorème de convergence dominée de Lebesgue,

$$
\begin{aligned}
\lim _{n \rightarrow+\infty} \int_{\Omega} S_{n}(u(T, x)) \mathrm{d} x & =\int_{\Omega} u(T, x) \mathrm{d} x, \\
\lim _{n \rightarrow+\infty} \int_{\Omega} S_{n}\left(u_{0}(x)\right) \mathrm{d} x & =\int_{\Omega} u_{0}(x) \mathrm{d} x .
\end{aligned}
$$

Ainsi,

$$
\lim _{n \rightarrow+\infty} \int_{Q}\{\mathcal{A}(x, u) \nabla \phi(u)\} \cdot \nabla S_{n}^{\prime}(u) \mathrm{d} x \mathrm{~d} t=\int_{\Omega}\left(u_{0}(x)-u(T, x)\right) \mathrm{d} x .
$$


De plus, en prenant $v=1_{\Omega}$ comme fonction-test dans (P), on obtient évidemment la relation de conservation de la masse globale

$$
\int_{\Omega}\left(u_{0}(x)-u(T, x)\right) \mathrm{d} x=0,
$$

d'où la relation $(\mathrm{L})$.

Remarque. - (L) peut s'écrire aussi

$$
\lim _{n \rightarrow+\infty} n \int_{Q \cap\{1 / 2 n \leq u \leq 1 / n\}} \phi^{\prime}(u)\{\mathcal{A}(x, u) \nabla u\} \cdot \nabla u \mathrm{~d} x \mathrm{~d} t=0
$$

ou encore

$$
L^{1}(Q)-\lim _{n \rightarrow+\infty} n \chi_{[1 / 2 n \leq u \leq 1 / n]} \phi^{\prime}(u)\{\mathcal{A}(x, u) \nabla u\} \cdot \nabla u=0 .
$$

Réciproquement, on peut vérifier que la formulation du problème $\left(\mathrm{P}^{*}\right)$ associé à la relation (L) permet de retrouver la formulation du problème (P). Pour cela, on considère comme fonction-test dans $\left(\mathrm{P}^{*}\right), S=S_{n}$. On multiplie ensuite par une fonction $\varphi, \varphi \in \mathcal{D}(] 0, T[)$, positive et on intègre sur $] 0, T[$.

On peut remarquer qu'il est suffisant de prouver le résultat pour des fonctions-test $w$ positives, étant donné que $H^{1}(\Omega)$ est réticulé et que $w$ peut s'écrire $w=w^{+}-w^{-}$. Enfin, par utilisation du lemme d'intégration de F. Mignot [3] et par passage à la limite, quand $n \rightarrow+\infty$, via la relation (L), on est en mesure de conclure puisque $L^{\infty}(\Omega) \cap H^{1}(\Omega)$ est dense dans $H^{1}(\Omega)$.

Finalement, il y a donc équivalence entre le problème $(\mathrm{P})$ et le problème $\left(\mathrm{P}^{*}\right)$, ce dernier étant associé à la relation $(\mathrm{L})$.

\section{Remarques}

1) La formulation du problème $\left(\mathrm{P}^{*}\right)$ permet, dans un premier temps, de ne considérer que la région de parabolicité, c'est-à-dire là où $u$ ne s'annule pas, en introduisant au sens ensembliste, pour tout $n \in \mathbb{N}^{*}$, l'ensemble $\mathcal{L}^{N+1}$-mesurable $u^{-1}\{[1 / 2 n, 1]\}$.

2) La relation (L) permet ensuite, dans un deuxième temps, par une propriété de raccordement maîtrisé d'atteindre la région où le phénomène d'hyperbolicité se produit, c'est-à-dire sur l'ensemble $\mathcal{L}^{N+1}$-mesurable $u^{-1}(0)$, dont la frontière correspond à la notion de "front" en Mécanique des fluides. 
On démontre maintenant l'unicité de la solution du problème $(\mathrm{P})$.

On introduit deux instants arbitraires $t$ et $\tau$ de $] 0, T[$.

Soient $u$ et $\widehat{u}$ deux solutions éventuelles du problème et par abus de notation, on écrit :

$$
u(t, \tau, x)=u(t, x) \quad \text { et } \quad \widehat{u}(t, \tau, x)=\widehat{u}(\tau, x) .
$$

Soient $\xi \in \mathcal{D}^{+}(] 0, T[)$ et $\rho_{\delta}$ une suite régularisante, $\delta>0$, telle que :

$$
\rho_{\delta} \in \mathcal{D}(\mathbb{R}), \quad \rho_{\delta} \geq 0 \quad \text { et } \quad \int_{\mathbb{R}} \rho_{\delta}(x) \mathrm{d} x=1
$$

Posons

$$
\xi_{\delta}(t, \tau)=\xi\left(\frac{t+\tau}{2}\right) \rho_{\delta}\left(\frac{t-\tau}{2}\right)
$$

avec $\delta$ assez petit afin que $\xi_{\delta} \in \mathcal{D}(] 0, T\left[^{2}\right)$ ce qui est effectivement possible.

Pour tout $\varepsilon>0, p_{\varepsilon}$ désigne l'approximation lipschitzienne de $\operatorname{sign}_{0}^{+}$ définie par

$$
\forall r \in \mathbb{R}, \quad p_{\varepsilon}(r)=\min \left(\frac{r^{+}}{\varepsilon}, 1\right) .
$$

D'après la démonstration de (L), on sait que, pour presque tout $t$ et presque tout $\tau, S_{n}^{\prime}(u)$ et $S_{n}^{\prime}(\widehat{u})$ sont éléments de $H^{1}(\Omega) \cap L^{\infty}(\Omega)$ (ainsi que $S_{n}(u)$ et $S_{n}(\widehat{u})$ ). De plus, d'après le lemme de G. Stampacchia, $p_{\varepsilon}\left(S_{n}(u)-S_{n}(\widehat{u})\right)$ est élément de $L^{\infty}(\Omega) \cap H^{1}(\Omega)$, pour presque tout $t$ et tout $\tau$.

Les problèmes $(\mathrm{P})$ et $\left(\mathrm{P}^{*}\right)$, ce dernier associé à $(\mathrm{L})$, étant équivalents, il est légitime de considérer comme fonctions-test dans l'algèbre de Banach $L^{\infty}(\Omega) \cap H^{1}(\Omega)$ :

- $v=S_{n}^{\prime}(u) p_{\varepsilon}\left(S_{n}(u)-S_{n}(\widehat{u})\right)$ pour l'équation relative à $u$,

- $v=S_{n}^{\prime}(\widehat{u}) p_{\varepsilon}\left(S_{n}(u)-S_{n}(\widehat{u})\right)$ pour l'équation relative à $\widehat{u}$ dans la formulation du problème $(\mathrm{P})$,

ce qui revient à prendre $w=p_{\varepsilon}\left(S_{n}(u)-S_{n}(\widehat{u})\right)$ dans la formulation de $\left(\mathrm{P}^{*}\right)$, avec $S=S_{n}$. 
On remplace $v$ dans chaque équation par les fonctions-test précédemment introduites. On multiplie par $\xi_{\delta}$ et on intègre sur $] 0, T\left[{ }^{2}\right.$; par différence, on obtient l'égalité $(\mathrm{E})$ suivante :

$$
\begin{aligned}
0= & \int_{0}^{T} \int_{0}^{T}\left\langle\frac{\partial u}{\partial t}, S_{n}^{\prime}(u) p_{\varepsilon}\left(S_{n}(u)-S_{n}(\widehat{u})\right)\right\rangle_{V^{\prime}, V} \xi_{\delta} \mathrm{d} t \mathrm{~d} \tau+ \\
& -\int_{0}^{T} \int_{0}^{T}\left\langle\frac{\partial \widehat{u}}{\partial \tau}, S_{n}^{\prime}(\widehat{u}) p_{\varepsilon}\left(S_{n}(u)-S_{n}(\widehat{u})\right)\right\rangle_{V^{\prime}, V} \xi_{\delta} \mathrm{d} t \mathrm{~d} \tau+ \\
& +\int_{] 0, T{ }^{2} \times \Omega}\left\{\mathcal{A}(x, u) \nabla \phi(u) S_{n}^{\prime}(u)-\mathcal{A}(x, \widehat{u}) \nabla \phi(\widehat{u}) S_{n}^{\prime}(\widehat{u})\right\} . \\
& \cdot \nabla p_{\varepsilon}\left(S_{n}(u)-S_{n}(\widehat{u})\right) \xi_{\delta} \mathrm{d} x \mathrm{~d} t \mathrm{~d} \tau+ \\
& +\int_{j 0, T{ }^{2} \times \Omega}\left(\{\mathcal{A}(x, u) \nabla \phi(u)\} \cdot \nabla S_{n}^{\prime}(u)-\{\mathcal{A}(x, \widehat{u}) \nabla \phi(\widehat{u})\} \cdot \nabla S_{n}^{\prime}(\widehat{u})\right) \\
& \times p_{\varepsilon}\left(S_{n}(u)-S_{n}(\widehat{u})\right) \xi_{\delta} \mathrm{d} x \mathrm{~d} t \mathrm{~d} \tau .
\end{aligned}
$$

On va passer à la limite dans chaque terme de l'expression (E), selon la stratégie suivante :

- la première étape consiste à faire tendre $\varepsilon$ vers $0^{+}, n$ étant fixé dans $\mathbb{N}^{*}$

- la deuxième étape qui prend en compte le phénomène hyperbolique marque le passage à la limite quand $n \rightarrow+\infty$;

- enfin, on fait tendre $\delta \rightarrow 0^{+}$.

Passage à la limite, quand $\varepsilon \rightarrow 0^{+}$

Considérons d'abord

$$
A_{\varepsilon}^{n}=\int_{10, T\left[^{2} \times \Omega\right.}\{\mathcal{A}(x, u) \nabla \phi(u)\} \cdot \nabla S_{n}^{\prime}(u) p_{\varepsilon}\left(S_{n}(u)-S_{n}(\widehat{u})\right) \xi_{\delta} \mathrm{d} x \mathrm{~d} t \mathrm{~d} \tau
$$

avec $n$ fixé. Or $\mathcal{L}^{N+2}$-presque partout dans $] 0, T\left[^{2} \times \Omega\right.$, on note que

$$
\begin{aligned}
& \lim _{\varepsilon \rightarrow 0^{+}}\{\mathcal{A}(x, u) \nabla \phi(u)\} \cdot \nabla S_{n}^{\prime}(u) p_{\varepsilon}\left(S_{n}(u)-S_{n}(\widehat{u})\right) \xi_{\delta}= \\
& =\{\mathcal{A}(x, u) \nabla \phi(u)\} \cdot \nabla S_{n}^{\prime}(u) \operatorname{sign}_{0}^{+}\left(S_{n}(u)-S_{n}(\widehat{u})\right) \xi_{\delta}
\end{aligned}
$$

et

$$
\left|\{\mathcal{A}(x, u) \nabla \phi(u)\} \cdot \nabla S_{n}^{\prime}(u) p_{\varepsilon}\left(S_{n}(u)-S_{n}(\widehat{u})\right) \xi_{\delta}\right| \leq \ell|\nabla \phi(u)|\left|\nabla S_{n}^{\prime}(u)\right| \xi_{\delta},
$$

majorante $\mathcal{L}^{N+2}$-intégrable sur $] 0, T\left[^{2} \times \Omega\right.$. 
Solutions renormalisées pour des équations autonomes des milieux poreux

Par conséquent, d'après le théorème de convergence dominée de Lebesgue dans $] 0, T\left[{ }^{2} \times \Omega\right.$,

$$
\begin{aligned}
\lim _{\varepsilon \rightarrow 0^{+}} A_{\varepsilon}^{n} & =\int_{\{] 0, T\left[^{2} \times \Omega\right\} \cap\left\{S_{n}(u)>S_{n}(\hat{u})\right\}}\{\mathcal{A}(x, u) \nabla \phi(u)\} \cdot \nabla S_{n}^{\prime}(u) \xi_{\delta} \mathrm{d} x \mathrm{~d} t \mathrm{~d} \tau \\
& =A^{n} .
\end{aligned}
$$

Passage à la limite, quand $n \rightarrow+\infty$, dans $A^{n}$

D'après la propriété (L) qui joue ici un rôle essentiel, on a :

$$
\{\mathcal{A}(x, u) \nabla \phi(u)\} \cdot \nabla S_{n}^{\prime}(u) \underset{n \rightarrow+\infty}{\longrightarrow} 0 \text { dans } L^{1}(Q) .
$$

Observant que la fonction $\xi_{\delta}$ est bornée dans $] 0, T\left[{ }^{2} \times \Omega\right.$, on obtient alors

$$
\lim _{n \rightarrow+\infty} A^{n}=0 .
$$

Par le même raisonnement, il vient :

$$
\begin{aligned}
\lim _{n \rightarrow+\infty}\left(\lim _{\varepsilon \rightarrow 0^{+}} \int_{10, T\left[^{2} \times \Omega\right.}\{\mathcal{A}(x, \widehat{u}) \nabla \phi(\widehat{u})\} \cdot \nabla S_{n}^{\prime}(\widehat{u}) \times\right. \\
\left.\times p_{\varepsilon}\left(S_{n}(u)-S_{n}(\widehat{u})\right) \xi_{\delta} \mathrm{d} x \mathrm{~d} t \mathrm{~d} \tau\right)=0 .
\end{aligned}
$$

Passage à la limite, $\varepsilon \rightarrow 0^{+}$(n fixé), dans

$$
\begin{array}{r}
B_{\varepsilon}^{n}=\int_{] 0, T\left[^{2} \times \Omega\right.}\left\{\mathcal{A}(x, u) \nabla \phi(u) S_{n}^{\prime}(u)-\mathcal{A}(x, \widehat{u}) \nabla \phi(\widehat{u}) S_{n}^{\prime}(\widehat{u})\right\} \\
\cdot \nabla p_{\varepsilon}\left(S_{n}(u)-S_{n}(\widehat{u})\right) \xi_{\delta} \mathrm{d} x \mathrm{~d} t \mathrm{~d} \tau
\end{array}
$$

D'après le résultat classique de $G$. Stampacchia, $\mathcal{L}^{2}$-presque partout sur ] $0, T\left[{ }^{2}\right.$ et $\mathcal{L}^{N}$-presque partout dans $\Omega$, on dispose de la règle de dérivation

$$
\begin{aligned}
\nabla & p_{\varepsilon}\left(S_{n}(u)-S_{n}(\widehat{u})\right)= \\
= & \frac{1}{\varepsilon} \nabla\left(S_{n}(u)-S_{n}(\widehat{u})\right) \chi_{\left[0<S_{n}(u)-S_{n}(\hat{u})<\varepsilon\right]} \\
=\frac{1}{\varepsilon} \nabla\left(S_{n}(u)-S_{n}(\widehat{u})\right) \chi_{\left[0<S_{n}(u)-S_{n}(\hat{u})<\varepsilon\right] \cap\left\{\left[S_{n}(\hat{u})>0\right] \cup\left[S_{n}(\hat{u})=0\right]\right\}} & \quad-737-
\end{aligned}
$$




\section{Anne Plouvier-Debaigt}

Introduisant alors pour tout $\varepsilon>0$, les deux ensembles $\mathcal{L}^{N+2}$-mesurables de ]0, $T\left[^{2} \times \Omega\right.$

$$
I_{\varepsilon}=\{] 0, T\left[^{2} \times \Omega\right\} \cap\left[0<S_{n}(u)-S_{n}(\widehat{u})<\varepsilon\right] \cap\left[S_{n}(\widehat{u})=0\right]
$$

et

$$
J_{\varepsilon}=\{] 0, T\left[^{2} \times \Omega\right\} \cap\left[0<S_{n}(u)-S_{n}(\widehat{u})<\varepsilon\right] \cap\left[S_{n}(\widehat{u})>0\right],
$$

on considère la décomposition

$$
\begin{array}{r}
B_{\varepsilon}^{n}=\frac{1}{\varepsilon} \int_{I_{\varepsilon}}\{\mathcal{A}(x, u) \nabla \phi(u)\} \cdot \nabla S_{n}(u) S_{n}^{\prime}(u) \xi_{\delta} \mathrm{d} x \mathrm{~d} t \mathrm{~d} \tau+ \\
+\frac{1}{\varepsilon} \int_{J_{\varepsilon}}\left\{\mathcal{A}(x, u) \nabla \phi(u) S_{n}^{\prime}(u)-\mathcal{A}(x, \widehat{u}) \nabla \phi(\widehat{u}) S_{n}^{\prime}(\widehat{u})\right\} \\
\cdot \nabla\left(S_{n}(u)-S_{n}(\widehat{u})\right) \xi_{\delta} \mathrm{d} x \mathrm{~d} t \mathrm{~d} \tau
\end{array}
$$

c'est-à-dire, par notation, $B_{\varepsilon}^{n}=B_{\varepsilon, 1}^{n}+B_{\varepsilon, 2}^{n}$. Or, $\mathcal{L}^{N+2}$-presque partout sur $I_{\varepsilon}$ et $J_{\varepsilon}$, il est loisible d'écrire que

$$
\mathcal{A}(x, u) \nabla \phi(u) S_{n}^{\prime}(u)=\mathcal{A}(x, u) \nabla S_{n}(u) \phi^{\prime}(u) .
$$

On obtient donc

$$
B_{\varepsilon, 1}^{n}=\frac{1}{\varepsilon} \int_{I_{\varepsilon}}\left\{\mathcal{A}(x, u) \nabla S_{n}(u)\right\} \cdot \nabla S_{n}(u) \phi^{\prime}(u) \xi_{\delta} \mathrm{d} x \mathrm{~d} t \mathrm{~d} \tau .
$$

Ainsi, en utilisant (H1)(ii), il s'ensuit que

$$
B_{\varepsilon, 1}^{n} \geq \frac{\alpha_{0}}{\varepsilon} \int_{I_{\varepsilon}}\left|\nabla S_{n}(u)\right|^{2} \phi^{\prime}(u) \xi_{\delta} \mathrm{d} x \mathrm{~d} t \mathrm{~d} \tau
$$

car $\phi^{\prime}$ et $\xi_{\delta}$ sont non négatives. D'où, $B_{\varepsilon, 1}^{n} \geq 0$, pour tout $\varepsilon>0$ et pour tout $n \in \mathbb{N}^{*}$. Par ailleurs,

$$
\begin{aligned}
& B_{\varepsilon, 2}^{n}= \frac{1}{\varepsilon} \int_{J_{\varepsilon}}\left\{\mathcal{A}(x, u) \nabla S_{n}(u) \phi^{\prime}(u)-\mathcal{A}(x, \widehat{u}) \nabla S_{n}(\widehat{u}) \phi^{\prime}(\widehat{u})\right\} \\
& \cdot \nabla\left(S_{n}(u)-S_{n}(\widehat{u})\right) \xi_{\delta} \mathrm{d} x \mathrm{~d} t \mathrm{~d} \tau \\
&=\frac{1}{\varepsilon} \int_{J_{\varepsilon}}\left\{\mathcal{A}(x, u) \nabla\left(S_{n}(u)-S_{n}(\widehat{u})\right)\right\} \\
&+\nabla\left(S_{n}(u)-S_{n}(\widehat{u})\right) \phi^{\prime}(u) \xi_{\delta} \mathrm{d} x \mathrm{~d} t \mathrm{~d} \tau+ \\
&+\frac{1}{\varepsilon} \int_{J_{\varepsilon}}\left\{\left(\mathcal{A}(x, u) \phi^{\prime}(u)-\mathcal{A}(x, \widehat{u}) \phi^{\prime}(\widehat{u})\right) \nabla S_{n}(\widehat{u})\right\} \\
& \cdot \nabla\left(S_{n}(u)-S_{n}(\widehat{u})\right) \xi_{\delta} \mathrm{d} x \mathrm{~d} t \mathrm{~d} \tau \\
&-738-
\end{aligned}
$$


Solutions renormalisées pour des équations autonomes des milieux poreux

En utilisant (H1)(ii), on a :

$$
\begin{array}{r}
B_{\varepsilon, 2}^{n} \geq \frac{\alpha_{0}}{\varepsilon} \int_{J_{\varepsilon}}\left|\nabla\left(S_{n}(u)-S_{n}(\widehat{u})\right)\right|^{2} \phi^{\prime}(u) \xi_{\delta} \mathrm{d} x \mathrm{~d} t \mathrm{~d} \tau+ \\
+\frac{1}{\varepsilon} \int_{J_{\varepsilon}}\left\{\left(\mathcal{A}(x, u) \phi^{\prime}(u)-\mathcal{A}(x, \widehat{u}) \phi^{\prime}(\widehat{u})\right) \nabla S_{n}(\widehat{u})\right\} \\
\cdot \nabla\left(S_{n}(u)-S_{n}(\widehat{u})\right) \xi_{\delta} \mathrm{d} x \mathrm{~d} t \mathrm{~d} \tau .
\end{array}
$$

Sur $J_{\varepsilon}$, on a en particulier $u \geq 1 / 2 n$.

De plus, la fonction $\phi^{\prime}$ s'annule uniquement en 0 et reste strictement positive sur ] 0,1$]$. Elle est en outre continue sur le compact $[1 / 2 n, 1]$ et admet donc un minimum, strictement positif d'après (H3), noté $m_{0, n}$, où

$$
m_{0, n}=\min _{r \in[1 / 2 n, 1]} \phi^{\prime}(r) \leq \phi^{\prime}(u) \text { sur } J_{\varepsilon} .
$$

Il vient donc (on est dans le cas où $u>\widehat{u}>1 / 2 n$ ) :

$$
\begin{aligned}
& B_{\varepsilon, 2}^{n} \geq \frac{\alpha_{0} m_{0, n}}{\varepsilon} \int_{J_{\varepsilon}}\left|\nabla\left(S_{n}(u)-S_{n}(\widehat{u})\right)\right|^{2} \xi_{\delta} \mathrm{d} x \mathrm{~d} t \mathrm{~d} \tau+ \\
&+\frac{1}{\varepsilon} \int_{J_{\varepsilon}}\left\{\mathcal{A}\left(x, \widetilde{S}_{n}^{-1}\left(\widetilde{S}_{n}(u)\right)\right) \phi_{0}^{\prime} \widetilde{S}_{n}^{-1}\left(\widetilde{S}_{n}(u)\right) \nabla S_{n}(\widehat{u})\right\} \\
& \cdot \nabla\left(S_{n}(u)-S_{n}(\widehat{u})\right) \xi_{\delta} \mathrm{d} x \mathrm{~d} t \mathrm{~d} \tau+ \\
&-\frac{1}{\varepsilon} \int_{J_{\varepsilon}}\left\{\mathcal{A}\left(x, \widetilde{S}_{n}^{-1}\left(\widetilde{S}_{n}(\widehat{u})\right)\right) \phi_{0}^{\prime} \widetilde{S}_{n}^{-1}\left(\widetilde{S}_{n}(\widehat{u})\right) \nabla S_{n}(\widehat{u})\right\} . \\
& \cdot \nabla\left(S_{n}(u)-S_{n}(\widehat{u})\right) \xi_{\delta} \mathrm{d} x \mathrm{~d} t \mathrm{~d} \tau,
\end{aligned}
$$

où $\widetilde{S}_{n}$ désigne la restriction de $S_{n}$ sur l'intervalle $[1 / 2 n, 1]$.

On vérifie que $\widetilde{S}_{n}^{-1}$ est höldérienne d'ordre $1 / 2$, puisque

$$
\forall y \in\left[0, \widetilde{S}_{n}(1)\right], \quad \widetilde{S}_{n}^{-1}(y)=\frac{\sqrt{y}}{\sqrt{n}}+\frac{1}{2 n} .
$$

De plus, on s'assure aussi que l'application $T_{n}$ définie par

$$
T_{n}: r \longrightarrow a_{i j}\left(\cdot, \widetilde{S}_{n}^{-1}(r)\right) \phi_{0}^{\prime} \widetilde{S}_{n}^{-1}(r), \quad r \in\left[0, \widetilde{S}_{n}(1)\right]
$$

est höldérienne d'ordre $1 / 2$ pour tout couple $(i, j) \in(1, \ldots, N)^{2}$ sur $\left[0, \widetilde{S}_{n}(1)\right]$, et de constante notée $c_{T_{n}}$, par le fait que, conformément à (H3), la restriction de $\phi^{\prime}$ à l'intervalle $[1 / 2 n, 1]$ est lipschitzienne. 


\section{Anne Plouvier-Debaigt}

En utilisant la norme matricielle subordonnée à la norme euclidienne de $\mathbb{R}^{N}$ notée $\|\cdot\|$, on peut alors écrire :

$$
\begin{aligned}
& \mid \frac{1}{\varepsilon} \int_{J_{\varepsilon}}\left\{\left(\mathcal{A}(x, u) \phi^{\prime}(u)-\mathcal{A}(x, \widehat{u}) \phi^{\prime}(\widehat{u})\right) \nabla S_{n}(\widehat{u})\right\} . \\
& \nabla\left(S_{n}(u)-S_{n}(\widehat{u})\right) \xi_{\delta} \mathrm{d} x \mathrm{~d} t \mathrm{~d} \tau \mid \leq \\
& \leq \frac{1}{\varepsilon} \int_{J_{\varepsilon}}\left\|\left|\mathcal{A}(x, u) \phi^{\prime}(u)-\mathcal{A}(x, \widehat{u}) \phi^{\prime}(\widehat{u}) \|\right| \nabla S_{n}(\widehat{u}) \mid \times\right. \\
& \times\left|\nabla\left(S_{n}(u)-S_{n}(\widehat{u})\right)\right| \xi_{\delta} \mathrm{d} x \mathrm{~d} t \mathrm{~d} \tau \\
& \leq \frac{\alpha_{0} m_{0, n}}{2 \varepsilon} \int_{J_{\varepsilon}}\left|\nabla\left(S_{n}(u)-S_{n}(\widehat{u})\right)\right|^{2} \xi_{\delta} \mathrm{d} x \mathrm{~d} t \mathrm{~d} \tau+ \\
& +\frac{c_{T_{n}}^{2}}{2 \alpha_{0} m_{0, n} \varepsilon} \int_{J_{\varepsilon}}\left|\nabla S_{n}(\widehat{u})\right|^{2}\left|S_{n}(u)-S_{n}(\widehat{u})\right| \xi_{\delta} \mathrm{d} x \mathrm{~d} t \mathrm{~d} \tau,
\end{aligned}
$$

d'après l'inégalité de Young et la propriété d'höldérianité précédemment démontrée

$$
\begin{gathered}
\leq \frac{\alpha_{0} m_{0, n}}{2 \varepsilon} \int_{J_{\varepsilon}}\left|\nabla\left(S_{n}(u)-S_{n}(\widehat{u})\right)\right|^{2} \xi_{\delta} \mathrm{d} x \mathrm{~d} t \mathrm{~d} \tau+ \\
+\frac{c_{T_{n}}^{2}}{2 \alpha_{0} m_{0, n}} \int_{J_{\varepsilon}}\left|\nabla S_{n}(\widehat{u})\right|^{2} \xi_{\delta} \mathrm{d} x \mathrm{~d} t \mathrm{~d} \tau .
\end{gathered}
$$

On étudie maintenant le deuxième terme du membre à droite de l'inégalité ci-dessus, le premier étant contrôlé par la minoration mise en évidence par le traitement de $B_{\varepsilon, 2}^{n}$.

$\mathcal{L}^{N+2}$-presque partout dans $] 0, T\left[^{2} \times \Omega\right.$, la fonction caractéristique $\chi_{\left[0<S_{n}(u)-S_{n}(\hat{u})<\varepsilon\right]}$ tend vers la fonction nulle, quand $\varepsilon \rightarrow 0^{+}, n$ fixé, $n \in \mathbb{N}^{*}$. De plus,

$$
\left.\left.\left|\chi_{\left[0<S_{n}(u)-S_{n}(\hat{u})<\varepsilon\right] \cap\left[S_{n}(\hat{u})>0\right]}\right| \nabla S_{n}(\hat{u})\right|^{2} \xi_{\delta}|\leq| \nabla S_{n}(\widehat{u})\right|^{2} \xi_{\delta},
$$

majorante de $L^{1}(] 0, T\left[^{2} \times \Omega\right)$. D'où, par l'utilisation du théorème de convergence dominée de Lebesgue,

$$
\lim _{\varepsilon \rightarrow 0^{+}} \int_{J_{\varepsilon}}\left|\nabla S_{n}(\widehat{u})\right|^{2} \xi_{\delta} \mathrm{d} x \mathrm{~d} t \mathrm{~d} \tau=0
$$


Solutions renormalisées pour des équations autonomes des milieux poreux

Il reste à étudier, pour presque tout $\tau \in] 0, T[$, le terme

$$
C_{\varepsilon}^{n}=\int_{0}^{T}\left\langle\frac{\partial u}{\partial t}, S_{n}^{\prime}(u) p_{\varepsilon}\left(S_{n}(u)-S_{n}(\widehat{u})\right)\right\rangle_{V^{\prime}, V} \xi_{\delta} \mathrm{d} t
$$

On constate que pour $\mathcal{L}^{N+1}$-presque tout $(\tau, x) \in Q$, la fonction définie sur $[0,1]$ par :

$$
r \longrightarrow S_{n}^{\prime}(r) p_{\varepsilon}\left(S_{n}(r)-S_{n}(\widehat{u})\right)
$$

est croissante et il est alors loisible d'appliquer le lemme de F. Mignot [3] fondé sur une double inégalité de convexité. Il s'ensuit que

$$
C_{\varepsilon}^{n}=-\int_{0}^{T} \frac{\partial \xi_{\delta}}{\partial t}\left(\int_{\Omega}\left\{\int_{\hat{u}}^{u} S_{n}^{\prime}(r) p_{\varepsilon}\left(S_{n}(r)-S_{n}(\widehat{u})\right) \mathrm{d} r\right\} \mathrm{d} x\right) \mathrm{d} t .
$$

Première étape : passage à la limite, quand $\varepsilon \rightarrow 0^{+}$( $n$ fixé)

On observe que pour tout $r \geq 0$ et $\mathcal{L}^{N+1}$-presque partout dans $Q$,

$$
\begin{aligned}
& S_{n}^{\prime}(r) p_{\varepsilon}\left(S_{n}(r)-S_{n}(\widehat{u})\right) \\
& \underset{\varepsilon \rightarrow 0^{+}}{\longrightarrow}\left\{\operatorname{sign}_{0}^{+}\left(S_{n}(r)-S_{n}(\widehat{u})\right)\right\} S_{n}^{\prime}(r)=\left\{\operatorname{sign}_{0}^{+}(r-\widehat{u})\right\} S_{n}^{\prime}(r),
\end{aligned}
$$

car, pour $r \leq 1 / 2 n$, les deux expressions sont nulles et pour $r>1 / 2 n, S_{n}$ est strictement croissante.

Cette dernière fonction étant bornée, il vient donc, par utilisation du théorème de convergence dominée de Lebesgue sur $[0,1]$, pour $\mathcal{L}^{N}$-presque tout $x \in \Omega$, et presque tout $\tau \in] 0, T[$,

$$
\lim _{\varepsilon \rightarrow 0^{+}} \int_{\hat{u}}^{u} S_{n}^{\prime}(r) p_{\varepsilon}\left(S_{n}(r)-S_{n}(\widehat{u})\right) \mathrm{d} r=\int_{\hat{u}}^{u} S_{n}^{\prime}(r) \operatorname{sign}_{0}^{+}(r-\widehat{u}) \mathrm{d} r .
$$

Ainsi, pour presque tout $\tau \in] 0, T[$,

$$
\lim _{\varepsilon \rightarrow 0^{+}} C_{\varepsilon}^{n}=-\int_{0}^{T} \frac{\partial \xi_{\delta}}{\partial t}\left(\int_{\Omega}\left\{\int_{\hat{u}}^{u} S_{n}^{\prime}(r) \operatorname{sign}_{0}^{+}(r-\widehat{u}) \mathrm{d} r\right\} \mathrm{d} x\right) \mathrm{d} t .
$$

De même, pour presque tout $t \in] 0, T[$,

$$
\begin{aligned}
& \lim _{\varepsilon \rightarrow 0^{+}} \int_{0}^{T}\left\langle\frac{\partial \widehat{u}}{\partial \tau}, S_{n}^{\prime}(\widehat{u}) p_{\varepsilon}\left(S_{n}(u)-S_{n}(\widehat{u})\right)\right\rangle_{V^{\prime}, V} \xi_{\delta} \mathrm{d} \tau= \\
& \quad=\lim _{\varepsilon \rightarrow 0^{+}} \int_{0}^{T} \frac{\partial \xi_{\delta}}{\partial \tau}\left(\int_{\Omega}\left\{\int_{\hat{u}}^{u} S_{n}^{\prime}(r) p_{\varepsilon}\left(S_{n}(u)-S_{n}(r)\right) \mathrm{d} r\right\} \mathrm{d} x\right) \mathrm{d} \tau \\
& =\int_{0}^{T} \frac{\partial \xi_{\delta}}{\partial \tau}\left(\int_{\Omega}\left\{\int_{\hat{u}}^{u} S_{n}^{\prime}(r) \operatorname{sign}_{0}^{+}(u-r) \mathrm{d} r\right\} \mathrm{d} x\right) \mathrm{d} t .
\end{aligned}
$$




\section{Anne Plouvier-Debaigt}

Deuxième étape : passage à la limite, quand $n \rightarrow+\infty$

Pour presque tout $\tau \in] 0, T$ [, le théorème de convergence dominée de Lebesgue permet d'affirmer que

$$
\int_{\hat{u}}^{u} S_{n}^{\prime}(r) \operatorname{sign}_{0}^{+}(r-\widehat{u}) \mathrm{d} r \underset{n \rightarrow+\infty}{\longrightarrow}(u-\widehat{u})^{+} \quad \mathcal{L}^{N+1} \text {-p.p. dans } Q
$$

et que, finalement, pour presque tout $\tau \in] 0, T[$,

$$
\begin{aligned}
& \lim _{n \rightarrow+\infty}\left(\lim _{\varepsilon \rightarrow 0^{+}} C_{\varepsilon}^{n}\right)= \\
& =\lim _{n \rightarrow+\infty}-\int_{0}^{T} \frac{\partial \xi_{\delta}}{\partial t}\left(\int_{\Omega}\left\{\int_{\hat{u}}^{u} S_{n}^{\prime}(r) \operatorname{sign}_{0}^{+}(r-\widehat{u}) \mathrm{d} r\right\} \mathrm{d} x\right) \mathrm{d} t . \\
& =-\int_{0}^{T} \frac{\partial \xi_{\delta}}{\partial t}\left(\int_{\Omega}(u-\widehat{u})^{+} \mathrm{d} x\right) \mathrm{d} t .
\end{aligned}
$$

En définitive, on retient que par une nouvelle application du théorème de convergence dominée sur $[0, T]$,

$$
\begin{aligned}
& \lim _{n \rightarrow+\infty}\left\{\lim _{\varepsilon \rightarrow 0^{+}} \int_{0}^{T} \int_{0}^{T}\left\langle\frac{\partial u}{\partial t}, S_{n}^{\prime}(u) p_{\varepsilon}\left(S_{n}(u)-S_{n}(\widehat{u})\right)\right\rangle_{V^{\prime}, V} \xi_{\delta}(t, \tau) \mathrm{d} t \mathrm{~d} \tau\right\}+ \\
& -\lim _{n \rightarrow+\infty}\left\{\lim _{\varepsilon \rightarrow 0^{+}} \int_{0}^{T} \int_{0}^{T}\left\langle\frac{\partial \widehat{u}}{\partial \tau}, S_{n}^{\prime}(\widehat{u}) p_{\varepsilon}\left(S_{n}(u)-S_{n}(\widehat{u})\right)\right\rangle_{V^{\prime}, V} \xi_{\delta}(t, \tau) \mathrm{d} t \mathrm{~d} \tau\right\} \\
& =-\int_{0}^{T} \int_{0}^{T} \int_{\Omega}\left(\frac{\partial \xi_{\delta}}{\partial t}+\frac{\partial \xi_{\delta}}{\partial \tau}\right)(u(t, x)-\widehat{u}(\tau, x))^{+} \mathrm{d} x \mathrm{~d} t \mathrm{~d} \tau \\
& =-\int_{0}^{T} \int_{0}^{T} \xi^{\prime}\left(\frac{t+\tau}{2}\right) \rho_{\delta}\left(\frac{t-\tau}{2}\right)\left(\int_{\Omega}(u(t, x)-\widehat{u}(\tau, x))^{+} \mathrm{d} x\right) \mathrm{d} t \mathrm{~d} \tau
\end{aligned}
$$

par définition de $\xi_{\delta}$ et le théorème de Fubini.

Ainsi, les passages à la limite dans chaque terme de l'égalité (E) conduisent à l'inégalité suivante :

$$
-\int_{0}^{T} \int_{0}^{T} \xi^{\prime}\left(\frac{t+\tau}{2}\right) \rho_{\delta}\left(\frac{t-\tau}{2}\right)\left(\int_{\Omega}(u(t, x)-\widehat{u}(\tau, x))^{+} \mathrm{d} x\right) \mathrm{d} t \mathrm{~d} \tau \leq 0 .
$$

Grâce à la notion de point de Lebesgue, il en résulte, lorsque $\delta \rightarrow 0^{+}$, que

$$
\forall \xi \in \mathcal{D}^{+}(] 0, T[), \quad-\int_{0}^{T} \xi^{\prime}(t)\left(\int_{\Omega}(u(t, x)-\widehat{u}(t, x))^{+} \mathrm{d} x\right) \mathrm{d} t \leq 0,
$$


Solutions renormalisées pour des équations autonomes des milieux poreux

c'est-à-dire

$$
\frac{\partial}{\partial t}\left(\int_{\Omega}(u(t, x)-\widehat{u}(t, x))^{+} \mathrm{d} x\right) \leq 0
$$

au sens de $\mathcal{D}^{\prime}(] 0, T[)$ d'où résultent immédiatement l'unicité (cf. [7], [9]) et la propriété de continuité forte de la solution de $[0, T]$ à valeurs dans $L^{1}(\Omega)$ par le fait que la distribution sur $] 0, T[$

$$
\frac{\partial}{\partial t}\left(\int_{\Omega}(u(t, x)-\widehat{u}(t, x))^{+} \mathrm{d} x\right)
$$

est une mesure négative sur $] 0, T[$.

\section{Références bibliographiques}

[1] ANDRÉ (N.) .- Sur l'unicité de problèmes quasi linéaires elliptiques et de leurs approximations numériques, Thèse de l'Université de Metz (1993).

[2] Artola (M.) et Tartar (L.) .- Un résultat d'unicité pour une classe de problèmes paraboliques quasi linéaires, Ricerche di Matematica XLIV, $\mathrm{n}^{\circ} 2$ (1996), pp. 173-184.

[3] Bamberger (A.) .- Étude d'une équation doublement non linéaire, Rapport Interne $\mathrm{n}^{\circ} 4$ du Centre de Mathématiques Appliquées de l'École Polytechnique (1977).

[4] Blanchard (D.) et Redwane (H.) .- Solutions renormalisées d'équations paraboliques à deux non-linéarités, C. R. Acad. Sci. Paris, Série I, 319 (1994), pp. 831-835.

[5] Boccardo (L.), Giachetti (D.), Diaz (J. I.) et Murat (F.) .- Existence and Regularity of Renormalized Solutions for some Elliptic Problems involving Derivatives of Nonlinear Terms, Journal of Differential Equations 106, $\mathrm{n}^{\circ} 2$ (décembre 1993).

[6] Di Perna (R. J.) et Lions (P.-L.) .- On the Cauchy problem for Boltzmann equations : global existence and weak stability, Ann. of Math. (2) 130, $\mathrm{n}^{\circ} 2$ (1989), pp. 321-366.

[7] GagneuX (G.) et Madaune-Tort (M.) .- Analyse mathématique de modèles non linéaires de l'ingénierie pétrolière, Collection "Mathématiques et Applications", Springer-Verlag, 22 (1995).

[8] MURAT (F.) .- Équations elliptiques non linéaires avec second membre $L^{1}$ ou mesure, Comptes rendus du $26^{\text {ème }}$ Congrès National d'Analyse Numérique, les Karellis (juin 1994), pp. A12-A24.

[9] Plouvier (A.) . - Sur une classe de problèmes d'évolution quasi linéaires dégénérés, Revista Matemática de la Universidad Complutense de Madrid 8, $\mathrm{n}^{\circ} 1$ (1995) pp. 197-227.

[10] Plouvier-Debaigt (A.) .- Sur quelques classes de problèmes d'évolution quasi linéaires, Thèse de l'Université de Pau et des Pays de l'Adour (1995). 